PROCEEDINGS OF THE

AMERICAN MATHEMATICAL SOCIETY

Volume 124, Number 6, June 1996

\title{
QUASIDISKS AND THE ZYGMUND PROPERTY
}

\author{
ZHU LAIYI AND ZHONG LEFAN
}

(Communicated by Albert Baernstein II)

\begin{abstract}
In this paper, we obtain a new characterization of quasidisks by
\end{abstract} the Zygmund property.

\section{INTRODUCTION}

Suppose that $D$ is a proper subdomain of the finite complex plane $\mathbb{C}$. For $z \in \mathbb{C}$ and $0<r<\infty$, let $B(z, r)$ denote the open disk with center $z$ and radius $r$. For constant $M>0$ and $f(z)$ analytic in $D$, we say $f(z) \in M H_{2}^{t}$ if the inequality

$$
\left|f(z)-P_{1}(f, z)\right| \leq M \delta \log \frac{2 d}{\delta}
$$

holds for any $z_{1}, z_{2} \in D$ and $z \in D \cap\left[B\left(z_{1}, d\right) \cup B\left(z_{2}, d\right)\right]$, where $d=\left|z_{1}-z_{2}\right|$, $\delta=\min \left\{\left|z-z_{1}\right|,\left|z-z_{2}\right|\right\}$, and

$$
P_{1}(f, z)=\frac{z_{2}-z}{z_{2}-z_{1}} f\left(z_{1}\right)+\frac{z-z_{1}}{z_{2}-z_{1}} f\left(z_{2}\right) .
$$

Let $H_{2}^{t}=\bigcup_{M>0} M H_{2}^{t}$. By [1], in the case $D=\{z:|z|<1\}, H_{2}^{t}$ is the following well-known Zygmund's class $\Lambda_{*}$ :

$\Lambda_{*}=\left\{f(z)\right.$ analytic in $\left.D \sup _{|h| \leq t} \max _{\theta \in|0,2 \pi|}\left|f\left(e^{i(\theta+h)}\right)-2 f\left(e^{i \theta}\right)+f\left(e^{i(\theta-h)}\right)\right| \leq M_{f} t\right\}$.

Zygmund's class $\Lambda_{*}$ has many important applications in approximation theory.

A domain $D \subset \mathbb{C}$ is said to be an $(\alpha, \beta)$-John domain, $0<\alpha \leq \beta<\infty$, if there exists $z_{0} \in D$ such that every $z \in D$ can be joined to $z_{0}$ by a rectifiable curve $\gamma:[0, d] \rightarrow D$, satisfying:

$$
\begin{aligned}
& \text { (a) } \gamma(0)=z, \quad \gamma(d)=z_{0} \text {; } \\
& \text { (b) } d \leq \beta ; \\
& \text { (c) } \operatorname{dist}(\gamma(s), \partial D) \geq \alpha \frac{s}{d} \quad(0 \leq s \leq d),
\end{aligned}
$$

where $s$ is the arc-length parameter.

Received by the editors March 23, 1994 and, in revised form, September 27, 1994 and November 30, 1994.

1991 Mathematics Subject Classification. Primary 30EXX

This research was supported by the National Science Foundation of China.

(C)1996 American Mathematical Society 
A domain $D \subset \mathbb{C}$ is said to be an $(\alpha, \beta)$-uniform domain, if for each pair of points $z_{1}, z_{2} \in D, z_{1} \neq z_{2}$, there is an $\left(\alpha\left|z_{1}-z_{2}\right|, \beta\left|z_{1}, z_{2}\right|\right)$-John domain $G$ such that $z_{1}, z_{2} \in G \subset D$.

$D$ is said to be a $K$-quasidisk if it is the image of a disk or half-plane under a $K$-quasiconformal mapping $f: \widehat{\mathbb{C}} \rightarrow \widehat{\mathbb{C}}$, where $\widehat{\mathbb{C}}=\mathbb{C} \cup\{\infty\}$. By [5], we know that if $D$ is a $K$-quasidisk, then $D$ is an $(\alpha, \beta)$-uniform domain for constants $\alpha$ and $\beta$ that depend only on $K$.

Quasidisks were characterized in [3] and [4] by the Hardy-Littlewood property (only for unbounded domains) and in [2] by the Schwarzian univalence criterion. In 1992, we obtained the following theorem.

Theorem $\mathbf{Z}([6])$. Suppose that $D$ is a quasidisk in $\mathbb{C}$. Then necessary and sufficient conditions for $f(z) \in H_{2}^{t}$ are that $f(z)$ is analytic in $D$ and satisfies

$$
\left|f^{\prime \prime}(z)\right|=O\left(\operatorname{dist}(z, \partial D)^{-1}\right), \quad z \in D .
$$

The sketch of the proof is as follows.

Suppose that $f(z) \in H_{2}^{t}$. For any $z \in D$, let $0<r<\operatorname{dist}(z, \partial D) / 4$. Then $D_{r}=B(z, r) \subset D$. Choosing $z_{1}, z_{2} \in D_{r}$ with $\left|z_{1}-z_{2}\right|=2 r$,

$$
D_{r} \subset B\left(z_{1}, 2 r\right) \cup B\left(z_{2}, 2 r\right) \subset D
$$

and

$$
\overline{D_{r}} \backslash\left\{z_{1}, z_{2}\right\} \subset B\left(z_{1}, 2 r\right) \cup B\left(z_{2}, 2 r\right) .
$$

Setting

$$
P_{1}(f, z)=\frac{z_{2}-z}{z_{2}-z_{1}} f\left(z_{1}\right)+\frac{z-z_{1}}{z_{2}-z_{1}} f\left(z_{2}\right)
$$

we have

$$
f(z)-P_{1}(f, z)=\frac{1}{2 \pi i} \int_{\partial D_{r}} \frac{f(\zeta)-P_{1}(f, \zeta)}{\zeta-z} d \zeta .
$$

It follows that

$$
f^{\prime \prime}(z)=\frac{1}{\pi i} \int_{\partial D_{r}} \frac{f(\zeta)-P_{1}(f, \zeta)}{(\zeta-z)^{3}} d \zeta
$$

By (1.1) we have

$$
\left|f^{\prime \prime}(z)\right| \leq \frac{M_{f}}{\pi r^{3}} \int_{\partial D_{r}} \delta(\zeta) \log \frac{4 r}{\delta(\zeta)}|d \zeta|
$$

where $\delta(\zeta)=\min \left\{\left|\zeta-z_{1}\right|,\left|\zeta-z_{2}\right|\right\}$.

Set

$$
\zeta=z+r e^{i \theta}, \quad z_{k}=z+r e^{i \theta_{k}}, \quad k=1,2 .
$$

Then

so

$$
\delta(\zeta)=2 r \min \left\{\sin \frac{\theta-\theta_{k}}{2}\right\}
$$

$$
\left|f^{\prime \prime}(z)\right| \leq \frac{M_{f}}{\pi r^{3}} 16 r^{2} \int_{0}^{2 \pi} \sin \frac{\theta}{2} \log \frac{2}{\sin \frac{\theta}{2}} d \theta \leq C_{0} \frac{1}{r} .
$$

Taking $r=\operatorname{dist}(z, \partial D) / 8$ yields $(1.5)$. 
In order to prove the sufficiency, let $z_{1}, z_{2} \in D$ and $z_{1} \neq z_{2}$. For any $z \in$ $\left[B\left(z_{1}, h\right) \cup B\left(z_{2}, h\right)\right] \cap D$, let $\delta=\min \left\{\left|z_{1}-z\right|,\left|z_{2}-z\right|\right\}$. Since $D$ is an $(\alpha, \beta)$-uniform domain, for $k=1,2$ there exists an $\left(\alpha\left|z_{k}-z\right|, \beta\left|z_{k}, z\right|\right)$-John domain $D_{k} \subset D$ containing $z$ and $z_{k}$. Let $z_{k_{0}}$ be the point in the definition of the $\left(\alpha\left|z_{k}-z\right|, \beta\left|z_{k}, z\right|\right)$ John domain $D_{k}, \gamma_{1 k}$ the corresponding rectifiable arc joining $z$ to $z_{k 0}$, and $\gamma_{2 k}$ the arc joining $z_{k}$ to $z_{k 0}$. Then we have

$$
\begin{aligned}
f(z)-P_{1}(f, z)= & \frac{z_{2}-z}{z_{2}-z_{1}}\left[\int_{\gamma_{1 k}}(\zeta-z) f^{\prime \prime}(\zeta) d \zeta-\int_{\gamma_{2 k}}\left(\zeta-z_{1}\right) f^{\prime \prime}(\zeta) d \zeta\right] \\
& -\frac{z-z_{1}}{z_{2}-z_{1}}\left[\int_{\gamma_{2 k}}\left(\zeta-z_{2}\right) f^{\prime \prime}(\zeta) d \zeta-\int_{\gamma_{1 k}}(\zeta-z) f^{\prime \prime}(\zeta) d \zeta\right] \\
& +\frac{\left(z_{2}-z\right)\left(z-z_{1}\right)}{z_{2}-z_{1}}\left[f^{\prime}\left(z_{10}\right)-f^{\prime}\left(z_{20}\right)\right] \\
= & S_{1}+S_{2}+S_{3} .
\end{aligned}
$$

It is easy to see that

$$
S_{k}=O(\delta), \quad k=1,2 .
$$

We estimate $S_{3}$. By virtue of (c) in (1.4), we have

$$
\operatorname{dist}\left(z_{k 0}, \partial D\right) \geq \alpha\left|z-z_{k}\right|, \quad k=1,2 .
$$

In the case

$$
\left|z_{10}-z_{20}\right| \leq \max _{k=1,2} \operatorname{dist}\left(z_{k 0}, \partial D\right) / 2,
$$

we may assume without loss of generality that $\operatorname{dist}\left(z_{20}, \partial D\right) \geq \operatorname{dist}\left(z_{10}, \partial D\right)$. Then the open disk $B_{2}$ with center $z_{20}$ and radius $\operatorname{dist}\left(z_{20}, \partial D\right) / 2$ is contained in $D$. Consequently, the distance of each point on $\bar{B}_{2}$ to $\partial D$ is not less than $\operatorname{dist}\left(z_{20}, \partial D\right) / 2$. Noting that $z_{10} \in \bar{B}_{2}$, let $\sigma$ be the segment from $z_{10}$ to $z_{20}$. Then we have

$$
\left|f^{\prime}\left(z_{10}\right)-f^{\prime}\left(z_{20}\right)\right|=\left|\int_{\sigma} f^{\prime \prime}(\zeta) d \zeta\right|=O(1)
$$

Thus

$$
S_{3}=O(\delta)
$$

In the case $\left|z_{10}-z_{20}\right| \geq \max _{k=1,2} \operatorname{dist}\left(z_{k 0}, \partial D\right) / 2$, it is not too difficult to show that

$$
\left|f^{\prime}\left(z_{10}\right)-f^{\prime}\left(z_{20}\right)\right|=O\left(\log \frac{\left|z_{10}-z_{20}\right|}{\delta}\right) .
$$

It follows from (1.13) and (1.12) that

$$
\left|f(z)-P_{1}(f, z)\right| \leq M \delta \log \frac{2 h}{\delta} .
$$

This proves that $f(z) \in H_{2}^{t}$.

Definition 1. Suppose that $D$ is a proper subdomain of $\mathbb{C}$. We say that $D$ has the Zygmund property if there exists a constant $M>0$ such that $f(z) \in H_{2}^{t}$ whenever $f(z)$ is analytic in $D$ and satisfies $\left|f^{\prime \prime}(z)\right| \leq M \operatorname{dist}(z, \partial D)^{-1}$ in $D$. 
From Theorem $\mathrm{Z}$ we know that quasidisks have the Zygmund property. In the present paper we show that, if an unbounded domain $\mathbb{C}$ has the Zygmund property, it is a quasidisk too. We thus obtain a characterization of unbounded quasidisks by the Zygmund property.

Theorem. Suppose that $D$ is a simply connected domain in $\widehat{\mathbb{C}}=\mathbb{C} \cup\{\infty\}$ with $\infty \in \partial D$ and that $D^{*}=\widehat{\mathbb{C}} \backslash \bar{D}$ is a domain. Then $D$ is a quasidisk if and only if both $D$ and $D^{*}$ have the Zygmund property.

\section{Some LEMmas}

Lemma $1([3])$. Suppose that $D$ is a simply connected subdomain of $\mathbb{C}$ and that $z_{0} \in \mathbb{C}$. If there exist points in $D \cap \bar{B}\left(z_{0}, r\right)$ which cannot be joined in $D \cap \bar{B}\left(z_{0}, b r\right)$, then there exist points $z_{1}, z_{2} \in D \cap \bar{B}\left(z_{0}, r\right)$ and $w_{0} \in \partial B\left(z_{0}, b r\right) \backslash D$ such that

$$
\left|h\left(z_{1}\right)-h\left(z_{2}\right)-2 \pi i\right| \leq \frac{2}{b-1}
$$

whenever $h(z)$ is an analytic branch of $\log \left(z-w_{0}\right)$ in $D$.

Under the conditions of Lemma 1 , let $z_{0}, z_{1}, z_{2}$ and $w_{0}$ be points as indicated in its statements. Choose an arc $\gamma$ in $D$ from $z_{1}$ to $z_{2}$, and let $z^{\prime}$ be the first point at which $\gamma$ meets $\partial B\left(z_{1}, \frac{d}{2}\right)$ when $\gamma$ is traversed from $z_{1}$ to $z_{2}$, where $d=\left|z_{1}-z_{2}\right|$. Denote by $\gamma^{\prime}$ the subarc of $\gamma$ from $z_{1}$ to $z^{\prime}$.

Given $h(z)$, an analytic branch of $\log \left(z-w_{0}\right)$ in $D$, let $h_{0}(z)$ be the analytic branch of $\log \left(z-w_{0}\right)$ in $B\left(z_{0}, b r\right)$ satisfying

$$
h_{0}\left(z_{1}\right)=h\left(z_{1}\right) .
$$

If $\sigma$ is the segment from $z_{1}$ to $z_{2}$, then plainly

$$
\left|h_{0}\left(z_{2}\right)-h_{0}\left(z_{1}\right)\right|=\left|\int_{\sigma} h_{0}^{\prime}(z) d z\right| \leq \int_{\sigma} \frac{|d z|}{\left|z-w_{0}\right|} \leq \frac{2}{b-1} .
$$

Since $\gamma^{\prime} \subset D \cap B\left(z_{1}, \frac{d}{2}\right) \subset B\left(z_{0}, b r\right)$, we infer from $(2.2)$

$$
h\left(z^{\prime}\right)=h_{0}\left(z^{\prime}\right) .
$$

Both $h_{0}(z)$ and $h(z)$ are analytic branches of $\log \left(z-w_{0}\right)$ in some neighborhood of $z_{2}$; we thus have $h_{0}\left(z_{2}\right)-h\left(z_{2}\right)=2 k \pi i$. Using (2.1), (2.2) and (2.3) we conclude

$$
\begin{aligned}
\left|h_{0}\left(z_{2}\right)-h\left(z_{2}\right)-2 \pi i\right| & \leq \frac{4}{b-1} \leq \frac{4}{3} \\
h_{0}\left(z_{2}\right)-h\left(z_{2}\right) & =2 \pi i
\end{aligned}
$$

For $z \in B\left(z_{0}, b r\right)$, let $f_{0}(z)=\left(z-w_{0}\right) h_{0}(z)$.

Lemma 2. Under the conditions of Lemma 1 and with $z_{0}, z_{1}, z_{2}, w_{0}$ and $z^{\prime}$ as indicated in the statement of the lemma and the ensuing discussion, it is the case that

$$
\left|f_{0}\left(z^{\prime}\right)-P_{1}\left(f_{0}, z^{\prime}\right)\right| \leq \frac{3 d}{b-2}
$$


Proof. Let $\sigma_{1}$ be the segment from $z_{1}$ to $z^{\prime}$, and $\sigma_{2}$ the segment from $z_{2}$ to $z^{\prime}$. We compute

$$
\begin{aligned}
\mid f_{0}\left(z^{\prime}\right) & -P_{1}\left(f_{0}, z^{\prime}\right) \mid \\
= & \left|\frac{z_{2}-z^{\prime}}{z_{2}-z_{1}}\left[f_{0}\left(z^{\prime}\right)-f_{0}\left(z_{1}\right)\right]+\frac{z^{\prime}-z_{1}}{z_{2}-z_{1}}\left[f_{0}\left(z^{\prime}\right)-f_{0}\left(z_{2}\right)\right]\right| \\
= & \left|\frac{z_{2}-z^{\prime}}{z_{2}-z_{1}} \int_{\sigma_{1}}\left(z-z_{1}\right) f_{0}^{\prime \prime}(z) d z+\frac{z^{\prime}-z_{1}}{z_{2}-z_{1}} \int_{\sigma_{2}}\left(z-z_{2}\right) f_{0}^{\prime \prime}(z) d z\right| \\
& \leq \frac{\left|z_{2}-z^{\prime}\right|}{\left|z_{2}-z_{1}\right|} \int_{\sigma_{1}}\left|z-z_{1}\right| \frac{|d z|}{\left|z-w_{0}\right|}+\frac{\left|z^{\prime}-z_{1}\right|}{\left|z_{2}-z_{1}\right|} \int_{\sigma_{2}}\left|z-z_{2}\right| \frac{|d z|}{\left|z-w_{0}\right|} \\
& \leq \frac{3}{2}\left|z_{1}-z^{\prime}\right| \frac{\left|z_{1}-z^{\prime}\right|}{(b-2) r}+\frac{\left|z_{1}-z^{\prime}\right|\left|z^{\prime}-z_{2}\right|}{\left|z_{2}-z_{1}\right|} \frac{z_{2}-z^{\prime} \mid}{(b-2) r} \\
& \leq \frac{6}{b-2}\left|z_{1}-z^{\prime}\right| \leq \frac{3 d}{b-2} .
\end{aligned}
$$

Definition $2([2])$. A set $E$ in $\widehat{\mathbb{C}}$ is said to be $a$-locally connected if for all $z_{0} \in \mathbb{C}$ and $r>0$, any pair of points in $E \cap \bar{B}\left(z_{0}, r\right)$ can be joined in $E \cap \bar{B}\left(z_{0}, a r\right)$ and any pair of points in $E \backslash B\left(z_{0}, r\right)$ can be joined in $E \backslash B\left(z_{0}, \frac{r}{a}\right)$.

Lemma $3([2])$. Suppose that a domain $D$ in $\mathbb{C}$ is a-locally connected and that $\partial D$ is connected and contains at least two points. Then $\partial D$ is a K-quasiconformal circle, where $K$ depends only on a.

\section{Proof of the Theorem}

The necessity of both $D$ and $D^{*}$ having the Zygmund property is ensured by Theorem Z. We must treat the sufficiency.

As in [2], we only need to prove the following proposition.

Proposition. Suppose that $D$ is a simply connected proper subdomain of $\mathbb{C}$ which has the Zygmund property. Then there exists a constant $b>4$, which depends only on the constant $M$ in Definition 1 , such that for all $z_{0} \in \mathbb{C}$ and $r>0$, each pair of points in $D \cap \bar{B}\left(z_{0}, r\right)$ can be joined in $D \cap \bar{B}\left(z_{0}, b r\right)$.

Proof. Choose

$$
b=\frac{M \log 4+8}{\pi}+2,
$$

and suppose the conclusion does not hold for some $z_{0} \in \mathbb{C}$ and $r>0$. Fix points $z_{1}, z_{2}$ in $D \cap \bar{B}\left(z_{0}, r\right)$ which cannot be joined in $D \cap \bar{B}\left(z_{0}, b r\right)$, and let $z^{\prime}$ and $w_{0}$ be as in section 2 .

Consider $h(z)$ an analytic branch of $\log \left(z-w_{0}\right)$ in $D$, along with the function

$$
f(z)=\left(z-w_{0}\right) h(z) .
$$

Then $f(z)$ is analytic in $D$, where it satisfies

$$
\left|f^{\prime \prime}(z)\right|=\frac{1}{\left|z-w_{0}\right|} \leq \operatorname{dist}(z, \partial D)
$$


The hypothesis on $D$ implies that

$$
\left|f(z)-P_{1}(f, z)\right| \leq M \delta \log \frac{2 d}{\delta}
$$

holds for all $z \in D \cap\left[B\left(z_{1}, d\right) \cup B\left(z_{2}, d\right)\right]$. In particular, (3.4) holds for the point $z^{\prime} \in D \cap\left[B\left(z_{1}, d\right) \cup B\left(z_{2}, d\right)\right]$. Noting $\delta=\left|z^{\prime}-z_{1}\right|=\left|z^{\prime}-z_{2}\right|=\frac{d}{2}$, we have

$$
\left|f\left(z^{\prime}\right)-P_{1}\left(f, z^{\prime}\right)\right| \leq \frac{M d \log 4}{2} .
$$

Because $f\left(z_{1}\right)=f_{0}\left(z_{1}\right), f\left(z^{\prime}\right)=f_{0}\left(z^{\prime}\right)$ and $f\left(z_{2}\right)=f_{0}\left(z_{2}\right)+2 \pi i\left(z_{2}-w_{0}\right)$, we obtain

$$
\begin{aligned}
\left|f\left(z^{\prime}\right)-P_{1}\left(f, z^{\prime}\right)\right| & =\left|f_{0}\left(z^{\prime}\right)-P_{1}\left(f_{0}, z^{\prime}\right)+P_{1}\left(f-f_{0}, z^{\prime}\right)\right| \\
& \geq\left|\frac{z^{\prime}-z_{1}}{z_{2}-z_{1}}\left[f\left(z_{2}\right)-f_{0}\left(z_{2}\right)\right]\right|-\left|f_{0}\left(z^{\prime}\right)-P_{1}\left(f_{0}, z^{\prime}\right)\right| \\
& =\pi\left|z_{2}-w_{0}\right|-\left|f_{0}\left(z^{\prime}\right)-P_{1}\left(f_{0}, z^{\prime}\right)\right| .
\end{aligned}
$$

By (2.6) and (3.1), we conclude that

$$
\begin{aligned}
\left|f\left(z^{\prime}\right)-P_{1}\left(f, z^{\prime}\right)\right| & \geq \pi(b-2) r-\frac{3 d}{b-2} \\
& \geq(M \log 4+8) d-\frac{3 \pi d M}{\log 4+8} \\
& \geq M d \log 4,
\end{aligned}
$$

which contradicts (3.5).

\section{ACKNOWLEDGMENT}

The authors are very grateful to the referee for his helpful suggestions.

\section{REFERENCES}

1. V. K. Dzjadyk, Introduction to the theory of uniform approximation of functions by polynomials, Nauk, Moscow, 1977. (Russian) MR 58:29579

2. F. W. Gehring, Univalent functions and the Schwarzian derivative, Comment. Math. Helv. 52 (1977), 561-572. MR 56:15905

3. F. W Gehring and O. Martio, Quasidisks and the Hardy-Littlewood property, Complex Variables Theory Appl. 2 (1983), 67-78. MR 84k:30020

4. R. Kaufman and J. M. Wu, Distances and the Hardy-Littlewood property, Complex Variables Theory Appl. 4 (1984), 1-5. MR 86d:30031

5. O. Martio and J. Sarvas, Injectivity theorems in plane and space, Ann. Acad. Sci. Fenn. 4 (1979), 383-401. MR 81i:30039

6. L. Y. Zhu, Uniform domain and theorm of Zygmund, Kexue Tongbo, 37 (1992), 1153-1156. (Chinese)

Department of Information, People's University, Beijing, 100872, People's Republic OF CHINA

Department of Mathematics, Peking University, Beijing, 100871, People's Republic OF CHINA 